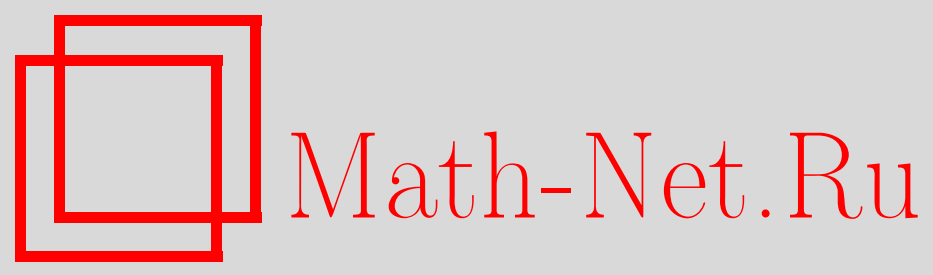

И. Г. Шандра, О геодезической подвижности римановых пространств, Матем. заметки, 2000, том 68, выпуск 4, 620626

DOI: https://doi.org/10.4213/mzm982

Использование Общероссийского математического портала Math-Net.Ru подразумевает, что вы прочитали и согласны с пользовательским соглашением http://www . mathnet.ru/rus/agreement

Параметры загрузки:

IP: 52.6 .47 .48

26 апреля 2023 г., 11:35:00

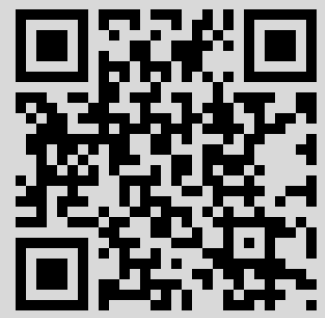




\section{О ГЕОДЕЗИЧЕСКОЙ ПОДВИЖНОСТИ РИМАНОВЫХ ПРОСТРАНСТВ}

\section{И.Г. Шандра}

В работе доказано, что степень геодезической подвижности $n$-мерного риманова пространства $(M, g)$, отличного от пространств постоянной кривизны, может принимать лиш значения $p=m(m+1) / 2+l$, где $m$ - число линейно независимых конциркулярных ковекторных полей на $M$, а $l$ изменяется от 1 до $[(n+1-m) / 3]$, где квадратные скобки означают целую часть числа. Тем самым, полностью решена задача об отыскании всех лакун в распределении степеней геодезической подвижности для данного класса пространств.

Библиографоия: 11 названий.

Введение. Задача о геодезическом отображении римановых пространств былавпервые сформулирована Леви-Чивитой [1], исследовавшим траекторную эквивалентность динамических систем. Понятие степени геодезической подвижности риманова пространства было введено Синюковым [2] как числовая характеристика мощности геодезического класса. Максимальную степень геодезической подвижности $p=(n+1)(n+2) / 2$ имеют $n$-мерные пространства постоянной кривизны и только они. Римановы пространства, у которьх степень подвижности больше 2 , являются с необходимостью пространствами $V(K)$, введенными в расмотрение Солодовниковым [3]. Синюковым была обнаружена лакунарность в распределении степеней геодезической подвижности подобно той, которая имеет место в теории движения. Им было доказано, что не существует псевдоримановых пространств, имеющих степень геодезической подвижности, заключенную в пределах

$$
\frac{(n-1) n}{2}+3<p<\frac{(n+1)(n+2)}{2} .
$$

Позже Микешем были определены точные гранищы первой лакуны [4]:

$$
\frac{(n-1)(n-2)}{2}+1<p<\frac{(n+1)(n+2)}{2}
$$

и выделена вторая лакуна. Метод нахождения таких лакун основывается на анализе условий интегрируемости основных уравнений геодезических отображений и связан со значительньми трудностями вычислительного характера. Поэтому, по всей видимости, до настоящего времени было известно лишь о двух лакунах. Примененный в настоящей работе принципиально иной подход позволил найти все лакуны в распределении степеней геодезической подвижности римановых пространств (пространств со знакоопределенной метрикой) и определиь их точные границы. Исследования ведутся локально, в классе достаточно гладких функций. 


\section{1. Предварительные сведения.}

1. Пусть $(M, g=\langle\cdot, \cdot\rangle)$ - $n$-мерное псевдориманово пространство, $\nabla$ - риманова связность, соответствующая метрике $g$, а $X, Y, Z$ - произвольные дифференцируемые векторные поля на $M$.

Отображение псевдориманова пространства $(M, g)$ на некотороепсевдориманово пространство $(\bar{M}, \bar{g})$ называется геодезическим, если при этом отображении каждая геодезическая пространства $(M, g)$ переходит в геодезическую пространства $(\bar{M}, \bar{g})$. Для того чтобы псевдориманово пространство $(M, g)$ допускало геодезическое отображение, необходимо и достаточно, чтобы на нем существовала симметрическая билинейная форма $A(X, Y)$, удовлетворяющая условиям [2]:

$$
\left(\nabla_{Z} A\right)(X, Y)=\lambda(X)\langle Y, Z\rangle+\lambda(Y)\langle X, Z\rangle
$$

при некотором градиентном ковекторе $\lambda$. В случае, когда $\lambda \neq 0$, геодезическое отображение назьвается нетривиальным (НГО). Если пространство $(M, g)(n>2)$ допускает два линейно независимых решения уравнений (1), не пропорциональных метрическому тензору, то имеют место следующие соотношения [4]:

$$
\begin{gathered}
\left(\nabla_{Z} \lambda\right)(X)=K A(X, Z)+\mu\langle X, Z\rangle, \\
\nabla_{Z} \mu=2 K \lambda(Z),
\end{gathered}
$$

где $K$ - некоторая константа, а $\mu$ - скалярное поле на $M$. При этом $(M, g)$ назьвают пространством $V(K)$ (см. [3], [4]).

Cтепенью геодезической подвижности р $n$-мерного псевдориманова пространства $(M, g)$ называется размерность линейного пространства решений системы (1). Любое псевдориманово пространство имеет степень геодезической подвижности не ниже 1 (так как на нем существует по крайней мере одно решение системы (1) вида $A=$ const $g$ ).

ЗАмЕчаниЕ 1. Как показано автором в [5], [6], если $K \neq 0$, то множество решений системы (1) пространства $V(K)$ образует йорданову алгебру $Q$ относительно операции умножения:

$$
(\stackrel{1}{A} * \stackrel{2}{A})(X, Y)=K(\langle\stackrel{1}{a} X, \stackrel{2}{a} Y\rangle+\langle\stackrel{2}{a} X, \stackrel{1}{a} Y\rangle)-(\stackrel{1}{\lambda} \otimes \stackrel{2}{\lambda}+\stackrel{2}{\lambda} \otimes \stackrel{1}{\lambda})(X, Y),
$$

где $\lambda$ - ковектор, соответствуюший решению $A$ системы $(1), a$ - линейньй оператор, определенный условиями $\langle a X, Y\rangle=A(X, Y)$.

Йорданову алгебру абсолютно параллельных симметрических билинейных форм на $(M, g)$ мы будем обозначать через $Q_{0}$. Очевидно, что $Q_{0} \subset Q$.

2. Ковекторное поле $\varphi$ на $M$ назьвается конциркулярным [6], если

$$
\left(\nabla_{Z} \varphi\right)(X)=\rho\langle X, Z\rangle,
$$

где $\rho$ - некоторое скалярное поле на $M$. Если к тому же имеет место условие

$$
\nabla_{Z} \rho=K \varphi(Z)
$$

где $K$ - некоторая константа, то конциркулярное ковекторное поле назьвается специальным. [7].

Линейное пространство конциркулярных ковекторных полей на $M$ обозначим через $\operatorname{Con}(M)$, а пространство ковариантно постоянных ковекторных полей на $M$ - через $\mathrm{Con}_{0}(M)$. 
ЗАмЕчАниЕ 2. Псевдоримановы пространства, допускающие конциркулярные основного типа поля, допускают НГО [2]. Так, если $\stackrel{1}{\varphi}, \ldots, \stackrel{m}{\varphi}$ - базис пространства $\operatorname{Con}(M)$, то тензорное поле

$$
A=\sum_{\alpha, \beta=1}^{m}{ }_{\alpha} \beta(\stackrel{\alpha}{\varphi} \otimes \stackrel{\beta}{\varphi}+\stackrel{\beta}{\varphi} \otimes \stackrel{\alpha}{\varphi})
$$

является решением системы $(1)$, где $\underset{\alpha}{C} \beta(=\underset{\beta}{C} \alpha)$ - некоторые константы. Это говорит о том, что степень подвижности такого пространства не ниже $m(m+1) / 2+1$ (для пространств, отличных от пространств постоянной кривизны). Множество решений системы (1), порожденных конциркулярными полями по формуле (4), образует идеал йордановой алгебры $Q[5]$.

ЗАмЕЧАниЕ 3 . Если $(M, g)$ допускает два или более линейно независимых конциркулярных ковекторных поля, то все они являются специальными, соответствуюшими одной и той же константе $K[7]$.

ЗАмЕчАниЕ 4. Псевдоримановы пространства, допускающие специальные конциркулярные основного типа ковекторные поля, являются пространствами $V(K)$. В частности, к пространствам $V(0)$ относятся пространства Широкова, т.е. псевдоримановы пространства, допускающие конциркулярное поле вида

$$
\left(\nabla_{Z} \varphi\right)(X)=\langle X, Z\rangle
$$

назьваемое сходящимся полем. Задание такого поля на $(n+1)$-мерном псевдоримановом пространстве $(\widetilde{M}, G)$ равносильно тому , что в специальной системе координат $\left(x^{0}, x^{i}\right)$ на $\widetilde{M}$ компоненты метрического тензора могут быть приведены к виду [8]

$$
G_{I J}=\exp \left(2 K x^{0}\right)\left(\begin{array}{cc}
-1 & 0 \\
0 & \frac{1}{K} g_{i j}
\end{array}\right)
$$

где $K(\neq 0)$ - некоторая константа, $g_{i j}\left(x^{k}\right)$ - компоненты метрического тензора некоторого $n$-мерного псевдориманова пространства $(M, g)$, отнесенного к системе координат $\left(x^{i}\right)$ (здесь и всюду в дальнейшем индексы $i, j, \ldots$ изменяются от 1 до $n$, а индексы $I, J, \ldots$ - от 0 до $n+1)$.

\section{2. Формулировка основного результата.}

Теорема. Степень геодезической подвижности $n$-мерного $(n>2)$ риманова пространства $(M, g)$, отличного от пространств постоянной кривизнь, мохет принимать лищь следующие значения:

$$
p=\frac{m(m+1)}{2}+l
$$

где $m=\operatorname{dim} \operatorname{Con}(M)$, a l изменяется от 1 до

$$
L=\left[\frac{n+1-m}{3}\right]
$$

(квадратные скобки означают иелую часть числа). 


\section{3. Вспомогательные утверждения.}

Лемма 1. Йорданова алгебра $Q$ n-мерного пространства $V(K)(K \neq 0)$ с метрическим тензором $g$ изоморфна йордановой алгебре $Q_{0}$ некоторого $(n+1)$-мерного пространства Широкова $(\widetilde{M}, G)$.

ДокАЗАТЕЛЬСтво. Путем прямых выкладок нетрудно убедится, что симметрическая билинейная форма $\widetilde{A}$, является абсолютно параллельной на $(n+1)$-мерном пространстве Широкова $(\widetilde{M}, G)$ тогда и только тогда, когда ее компоненты в специальной системе координат $\left(x^{0}, x^{i}\right)$ на $\widetilde{M}$ приводится к виду

$$
\widetilde{A}_{I J}=\exp \left(2 K x^{0}\right)\left(\begin{array}{cc}
\mu & \lambda_{i} \\
\lambda_{j} & A_{i j}
\end{array}\right),
$$

где $A_{i j}, \lambda_{i}, \mu$ - компоненты тензорных полей $A, \lambda, \mu$, являющихся решениями системы (1)-(3), в системе координат $\left(x^{i}\right)$ на $M$. Таким образом, соотношения (8) устанавливают взаимно однозначное соответствие между абсолютно параллельными квадратичными формами $(n+1)$-мерного пространства Широкова и квадратичными формами $n$-мерного пространства $V(K)(K \neq 0)$, являюшимися решениями системы (1).

ЗАмЕчАниЕ 5. Подобным образом нетрудно установить взаимно однозначное соответствие между абсолютно параллельными ковекторными полями $(n+1)$-мерного пространства Широкова и конциркулярными полями $n$-мерного пространства $V(K)(K \neq 0)$.

Лемма 2. Размерность алгебры $Q_{0}$ на $n$-мерном римановом пространстве Широкова, отличном от плоского, может принимать лишь следующие значения:

$$
\operatorname{dim} Q_{0}=\frac{k(k+1)}{2}+l,
$$

әде l изменяется от 1 до $L_{a}, k=\operatorname{dim} \operatorname{Con}_{0}(M), a$

$$
L_{a}=\left[\frac{n-k}{3}\right] \text {. }
$$

ДокАЗАТЕЛЬСтво. Пусть $B \in Q_{0}$. Если риманово пространство $(M, g)$ неприводимо, то, как известно [9], оно допускает такие поля только вида $B=$ const $g$. И в этом случае теорема доказана $(m=0, l=1)$. Если же $(M, g)$ приводимо, то метрическая форма $d s^{2}=g_{i j} d x^{i} d x^{j}$ и квадратичная форма $B=b_{i j} d x^{i} d x^{j}$ в некоторой системе координат $\left(x^{i}\right)$ на $M$ могут быть одновременно приведены к виду [9]

$$
\begin{aligned}
& d s^{2}=\stackrel{0}{g}_{i_{0} j_{0}} d x^{i_{0}} d x^{j_{0}}+\sum_{\alpha=1}^{l} \stackrel{\alpha}{g}_{i_{\alpha} j_{\alpha}}\left(x^{k_{\alpha}}\right) d x^{i_{\alpha}} d x^{j_{\alpha}}, \\
& B=C_{i_{0} j_{0}} d x^{i_{0}} d x^{j_{0}}+\sum_{\alpha=1}^{l} C_{\alpha}^{\alpha} g_{i_{\alpha} j_{\alpha}}\left(x^{k_{\alpha}}\right) d x^{i_{\alpha}} d x^{j_{\alpha}},
\end{aligned}
$$

где $C_{i_{0} j_{0}}=\left(C_{j_{0} i_{0}}\right), \underset{\alpha}{C}$ - некоторые константы, ${\stackrel{\alpha}{i_{\alpha} j_{\alpha}}}_{\alpha}\left(x^{k_{\alpha}}\right)$ - метрический тензор некоторого неприводимого риманового пространства $(\stackrel{\alpha}{M}, \stackrel{\alpha}{g}), \stackrel{0}{g} i_{i_{0} j_{0}}$ (= const) - метрический 
тензор плоского риманова пространства $\stackrel{0}{M}, \stackrel{0}{g}), \operatorname{dim} \stackrel{\alpha}{M}=n_{\alpha}, \operatorname{dim} \stackrel{0}{M}=n_{0}=k ;$ индексы $i_{0}, j_{0}, k_{0}, \ldots$ изменяются от 1 до $n_{0} ; i_{\alpha}, j_{\alpha}, k_{\alpha}, \ldots$ изменяются от $1+\sum_{\beta=0}^{\alpha-1} n_{\beta}$ до $\sum_{\beta=0}^{\alpha} n_{\beta} ; \sum_{\beta=0}^{l} n_{\beta}=n$. Так как исследуемое нами риманово пространство $(M, g)$ допускает сходящееся конциркулярное поле, то все пространства $(\stackrel{\alpha}{M}, \stackrel{\alpha}{g})$ будут также допускать такое поле; следовательно, должны иметь размерность не ниже 3, так как в противном случае они были бы плоскими, что противоречит их неприводимости. Поэтому величина $l$ в формуле (6) может принимать значения от 0 до [( $n-k) / 3]$. Причем случай $l=0$, как очевидно, соответствует плоскому пространству.

ЗАмЕчАниЕ 6 . Пусть $A \in Q_{0}$ и $\varphi$ - сходящееся поле на пространстве Широкова $(M, g)$. Тогда существует ковекторное поле $\psi$ на $M$ такое, что $A=L_{P} g$, где $P$ - векторное поле, сопряженное относительно метрики $g$ ковекторному полю $\psi$, а $L_{P}$ - производная Ли в направлении $P$. Нетрудно проверить, что таковьм является ковекторное поле $\psi(X)=\varphi(a X)$.

4. Доказательство основного результата. Всюду в дальнейшем, если это не оговорено особо, мы будем предполагать, что $(M, g)$ является римановым пространством (т.е. метрика $g$ положительно определена). Как уже было сказано выше, римановы пространства, допускающие степень геодезической подвижности $p>2$, т.е. имеющие не менее двух линейно независимых решений системы (1), не пропорциональных метрическому тензору, являются по необходимости пространствами $V(K)$. Рассмотрим два возможных случая.

1) Пусть $K=0$. Как показано в работе[10], пространства $V(0)$ со знакоопределенной метрикой являются пространствами Широкова. Пусть $A$ - произвольное решение уравнений $(1)$, a $\stackrel{1}{\varphi}, \ldots, \stackrel{m}{\varphi}$ - базис линейного пространства $\operatorname{Con}(M)$. Мы можем выбрать его таким образом, что $\stackrel{1}{\varphi}$ будет сходящимся полем, а $\stackrel{2}{\varphi}, \ldots, \stackrel{m}{\varphi}$ - ковариантно постоянными. Из соотношений (2) при $K=0$ следует, что ковектор $\lambda$, соответствующий $A$, является конциркулярным полем, поэтому

$$
\lambda=\sum_{\alpha=1}^{m} C_{\alpha}^{\alpha} .
$$

Тогда, как нетрудно проверить, тензор

$$
B=2 A-C_{1} \varphi^{1} \otimes \stackrel{1}{\varphi}-2 \sum_{\alpha=2}^{m} C_{\alpha}^{\alpha} \otimes \stackrel{1}{\varphi}
$$

будет ковариантно постоянным. Таким образом,

$$
p=\operatorname{dim} Q_{0}+\operatorname{dim} \operatorname{Con}(M) .
$$

Учитьвая, что $\operatorname{dim} \operatorname{Con}_{0}(M)=m-1$, из (12) и леммы 2 мы получаем утверждение нашей теоремы.

2) Пусть $K \neq 0$. Лемма 1 устанавливает взаимно однозначное соответствие между решениями системы (1) на $n$-мерном римановом пространстве $(M, g)$ и ковариантно постоянньпи квадратичньми формами на $(n+1)$-мерном пространстве $V(0)$ с метрическим тензором $G$, определяемым формулой $(5)$. Если $K<0$, то $G$ будет знакоопределена 
и к ней применимы рассуждения, использованные нами в п. 1. Поэтому, полагая в соотношениях (9) и (10) $k:=m, n:=n+1$, мы снова приходим к (6), (7). Если же $K>0$, то метрика $G$ имеет лоренцеву сигнатуру. Тогда одна из метрик $\stackrel{0}{G}, \stackrel{1}{G}, \ldots, \stackrel{l}{G}$ в разложении (11) для $G$ будет иметь лоренцеву сигнатуру, а остальные будут знакоопределены. Если таковой является $\stackrel{0}{G}$, то это ничего не изменяет по сравнению со случаем $K<0$. Пусть таковой является одна из метрик $\stackrel{\alpha}{G}$. Будем считать для определенности $\stackrel{1}{G}$. Как отмечалось вьше (см. замечание 6), пространства Широкова допускают симметрические абсолютно параллельные билинейные формы только лишь вида $A=L_{Z} g$. Все такие формы на неприводимом псевдоримановом пространстве $(\stackrel{1}{M}, \stackrel{1}{G})$ лоренцевой сигнатуры, как это показано Аминовой [11], определяются соотношениями

$$
L_{Z} g=\underset{1}{C} \stackrel{1}{G}+\underset{2}{C} \psi \otimes \psi
$$

где $\underset{1}{C}, \underset{2}{C}=$ const, а $\psi$ - ковариантно постоянное изотропное ковекторноеполе на $(\stackrel{1}{M}, \stackrel{1}{G})$. Учитьвая, что помимо поля $\varphi$ пространство $(\stackrel{1}{M}, \stackrel{1}{G})$ допускает еще и сходящееся конциркулярноеполе, его размерность должна быть не менее 4, так как в противном случае оно было бы плоским. С другой стороны, $\operatorname{dim} \stackrel{0}{M}=m-1$. Поэтому суммарно это не повлияет на оценку $l$ в разложении (11) для $G$. Таким образом, и в этом случае мы опять приходим к формулам (6), (7).

Непосредственно из доказанной теоремы в случае, когда $m=0$, мы получаем

СлЕДСтвИЕ. Римановы пространства $(n>2)$, не допускающие конииркулярных ковекторных полей, имеют степень геодезической подвижности, не превышающую $[(n+1) / 3]$.

ЗАмЕчАниЕ 7. Покажем, что существуют римановы пространства, для которых достигаются степени геодезической подвижности, указанные в (6), т.е. что границы лакун, определенные этой теоремой, являются точньми. Действительно, рассмотрим $\widehat{n}$-мерное риманово пространство Широкова $(\widehat{M}, \widehat{g})$, метрическая форма которого в некоторой системе координат на $\widehat{M}$ приводится к виду

$$
d \widehat{s}^{2}=\exp \left(2 x^{1}\right)\left(\left(d x^{1}\right)^{2}+\widetilde{g}_{i_{2} j_{2}}\left(x^{k_{2}}\right) d x^{i_{2}} d x^{j_{2}}\right)
$$

где $\widetilde{g}_{i_{2} j_{2}}\left(x^{k_{2}}\right)$ - метрический тензор некоторого $(\widehat{n}-1)$-мерного симметрического риманового пространства $(\widetilde{M}, \widetilde{g})$; индексы $i_{2}, j_{2}, k_{2}$ изменяются от 2 до $\widehat{n}$. Очевидно, что $(\widehat{M}, \widehat{g})$ может допускать абсолютно параллельные квадратичные формы только лишь вида $b=$ const $g$. Действительно, в противном случае на основании леммы $1(\widetilde{M}, \widetilde{g})$ допускало бы квадратичную форму, являющуюся решением системы (1) (т.е. допускало бы НГО), и мы пришли бы к противоречию, ведь, как известно [2], симметрические пространства не допускают НГО. Таким образом, если в соотношении (11) в качестве $\stackrel{\alpha}{g}$ выбрать метрики вида $(13)$, то степень геодезической подвижности $(M, g)$ будет равна $p=m(m+1) / 2+l$. 


\section{СПИСОК ЦИТИРОВАННОЙ ЛИТЕРАТУРЫ}

[1] Levi-Civita T. Sulle transformatzioni delle equazioni dinamiche // Annali Mat. 1896. V. 60. № 2. P. 255-300.

[2] Синюков Н. С. Геодезические отображения римановых пространств. М.: Наука, 1979.

[3] Солодовников А. С. Пространства с общими геодезическим // Тр. семинара по векторному и тензорному анализу. 1961. № XI. С. 43-102.

[4] Микеш Й. Геодезические отображения афффинносвязных и римановых пространств // Итоги науки и техн. ВИНИТИ. Тематические обзоры. Геометрия-2, 1994.

[5] Шандра И.Г. Пространства $V(K)$ и Йордановы алгебры // Тр. геом. семинара, посвященного памяти Лобачевского. Т. 1. Казань: КГУ, 1992. С. 99-104.

[6] Шандра И.Г. Геодезические отображения эквидистантных пространств и Йордановых алгебр пространства $V(K) / /$ Дифференциальная геометрия многообразий фигур. № 24 . Калининград: Калинингр. ун-т, 1993. С. 104-111.

[7] Fialkow A. Conformal geodesics // Trans. Amer. Math. Soc. 1939. V. 45. P. 443-473.

[8] Широков П.А. О сходящихся направлениях в римановых пространствах // Изв. физ.-матем. общества (3). Т. 7. Казань, 1934-1935. С. 77-88.

[9] Кручкович Г. И., Солодовников А. С. Постоянные симметрические тензоры римановых пространств // Изв. вузов. Матем. 1959. № 3. С. 147-158.

[10] Микеш Й. Об эквидистантных келеровых пространствах // Матем. заметки. 1985. Т. 38. № 4. С. $627-633$.

[11] Аминова А. В. Группы преобразований римановых пространств // Итоги науки и техн. ВИНИТИ. Проблемы геометрии. 1990. Т. 22. С. 97-165.

Финансовая академия при правительстве Российской Федерации

Поступило

E-mail : shandra@orc.ru

29.11.1999 